\title{
Selective ablation with UV lasers of a-Si:H thin film solar cells in direct scribing configuration
}

\author{
S. Lauzurica J.J. García-Ballesteros, M. Colina, I. Sánchez-Aniorte, C. Molpeceres
}

\begin{abstract}
A B S T R A C T
Monolithical series connection of silicon thin-film solar cells modules performed by laser scribing plays a very important role in the entire production of these devices. In the current laser process interconnection the two last steps are developed for a configuration of modules where the glass is essential as transparent substrate. In addition, the change of wavelength in the employed laser sources is sometimes enforced due to the nature of the different materials of the multilayer structure which make up the device. The aim of this work is to characterize the laser patterning involved in the monolithic interconnection process in a different configurations of processing than the usually performed with visible laser sources. To carry out this study, we use nanosecond and picosecond laser sources working at $355 \mathrm{~nm}$ of wavelength in order to achieve the selective ablation of the material from the film side. To assess this selective removal of material has been used EDX (energy dispersive using X-ray) analysis, electrical measurements and confocal profiles. In order to evaluate the damage in the silicon layer, Raman spectroscopy has been used for the last laser process step. Raman spectra gives information about the heat affected zone in the amorphous silicon structure through the crystalline fraction calculation. The use of ultrafast sources, such as picoseconds lasers, coupled with UV wavelength gives the possibility to consider materials and substrates different than currently used, making the process more efficient and easy to implement in production lines. This approach with UV laser sources working from the film side offers no restriction in the choice of materials which make up the devices and the possibility to opt for opaque substrates.
\end{abstract}

\section{Introduction}

Thin film a-Si:H solar cell technology has great potential to produce low cost modules. The cost reduction is determined by the scaling rate of the production capability, by means of the improvement of each step throughout the fabrication process. Regarding the monolithic interconnection process of the cells to form a module, laser-controlled ablation of individual layers is essential for the isolation and interconnection of the cells. Material ablation with minimum debris and small heat-affected zone with low processing cost, is one of the main challenges for the successful implementation of laser micromachining as a competitive technology in this field [2-5]. The three steps (P1, P2 and P3) for monolithic interconnection of solar cells within modules are nowadays defined by DPSS (diode pump solid state) laser sources working at $\operatorname{IR}(\lambda=1064 \mathrm{~nm})$ and VIS ( $\lambda=532 \mathrm{~nm}$ ) wavelengths for thin film module fabrication. These steps consist in removing the front contact layer usually a TCO (transparent conductive oxide) (P1), a-Si structure ablation (P2) and finally back contact removal (P3). It is important to high- light the back scribing configuration to carry out P2 and P3, where the laser attacks the material through the glass substrate, restricting the latter to be transparent to the laser radiation.

The laser process must be improved in order to minimize shunting problems which are closely related to the inherent thermal affection of laser irradiation at these wavelengths and nanosecond pulse duration. This work moves towards the possibility of performing the three laser steps with UV laser sources working at two different temporal ranges of pulse width (nanosecond and picosecond) in order to eliminate the thermal affectation and performing the process from the film side, which makes the process suitable for the two different configurations (superstrate and substrate) and other kinds of technologies (CIS/CIGS, CdTe) including opaque substrates [5]. Especially important is the picoseconds laser scribing evaluation, due to the lack of literature in this subject and the fact that these laser sources have become nowadays good industrial tools with high repetition rates, which allows the high processing speed required for industrial application.

This work presents the characterization of the three laser steps (P1, P2 and P3), focusing on the special difficulties of selective ablation from the film side of all the layers which make up these devices, in particular P2 and P3 steps, where the affectation of the silicon structure layer compromises the proper function of the same. In 
P2 a good control of energy is necessary to avoid damage on the layer underneath and the removed silicon may be redeposited on the edge of the grooves, leading to the formation of ridges that may affect the layer deposited on top. In P3, more important in terms of the behaviour of the final device, the back contact scribing may cause shunts between the layers, when the laser interacts with them due to the heat affectation. The change in the a-Si:H structure could lead to impoverishment of the electrical properties of the final module.

Laser ablation for the three steps will be discussed, focusing our study on the ablation threshold measurements and process-quality assessment by means of EDX, SEM images and Raman spectra in order to evaluate the heat affected zone in the amorphous silicon structure through the crystalline fraction calculation.

The success of this approach determines the suitability of the laser process on solar modules with opaque substrates. This leads to the possibility of using cheaper and more versatile materials. In previous works, we have demonstrated some of the advantages of using UV wavelengths for minimizing the thermal affectation, hence reducing the probability of shunting problems in the device $[6,7]$.

\section{Experimental procedure}

\subsection{Laser systems}

For the direct-scribing experiments two different laser sources, with pulse durations of nanosecond and picoseconds, have been used. The former is a DPSS laser operating at $355 \mathrm{~nm}$ wavelength (HIPPO SPECTRA PHYSICS), with pulse duration of $15 \mathrm{~ns}$, and the latter is a picosecond DPSS emitting at $355 \mathrm{~nm}$ wavelength and pulsewidth of $8 \mathrm{ps}$ (LUMERA SUPER RAPID). The typical pulse energy distribution of both sources is Gaussian. The power of both lasers is adjusted by an external attenuator with the maximum power set through the excitation current of the respective pump sources. The beams have been delivered through a scanner head in order to achieve high speed processing. VIS wavelengths of DPSS lasers have not been used, given the fact that this work does not consider back-scribing processes and is focussed only in direct writing from the film side.

\subsection{Sample preparation}

Two types of samples have been processed. For studying the ablation thresholds, single-layers of material have been deposited on glass. For the laser scribing study, non-finished solar cells were deposited for the different steps in the superstrate configuration (AZO/a-Si:H/ASAHI-U (TCO)/glass). The amorphous-silicon layers have been deposited by PECVD (plasma-enhanced chemical-vapor deposition) in an MVSystems capacitive-coupled reactor yielding highly uniform films with thicknesses in the range $500-600 \mathrm{~nm}$. For TCO single layer ablation we used commercial Asahi-U $\left(\mathrm{SnO}_{2}: \mathrm{F}\right)$ and in-house-prepared ITO $\left(\mathrm{SnO}_{2}: \mathrm{In}_{2} \mathrm{O}_{3}\right)$ and $\mathrm{AZO}(\mathrm{ZnO}: \mathrm{Al})$ samples were prepared in a RF sputtering plant using a MVSystems device.

\subsection{Measurement and characterization techniques}

Ablation-profile measurements and morphological characterization have been made using a confocal laser scanning microscope (CLSM) Leica ICM 1000. Additional SEM (scanning electron microscopy) (Hitachi S-3500N) and EDX (energy dispersive X-ray (spectroscopy)) (Röntec QX2) profile analysis images are included for a better comprehension of the morphology and selectiveablation process of the scribes. Combining these three techniques,
Table 1

Ablation threshold for thin-film materials $\left(\mathrm{J} / \mathrm{cm}^{2}\right)$.

\begin{tabular}{lll}
\hline Materials & $\phi_{\mathrm{th}}\left(\mathrm{J} / \mathrm{cm}^{2}\right)$ & \\
\cline { 2 - 3 } & $355 \mathrm{~nm} 15 \mathrm{~ns}$ & $355 \mathrm{~nm} \mathrm{8} \mathrm{ps}$ \\
\hline Asahi-U ${ }^{\circledR 2}\left(\mathrm{SnO}_{2}: \mathrm{F}\right)$ & 1.42 & 0.47 \\
$\mathrm{ITO}\left(\mathrm{SnO}_{2}: \mathrm{In}_{2} \mathrm{O}_{3}\right)$ & 0.21 & 0.36 \\
$\mathrm{ZnO}: \mathrm{Al}$ & 0.18 & 0.32 \\
a-Si:H & 0.12 & 0.22 \\
$\mathrm{Al}$ & 0.62 & 0.14 \\
\hline
\end{tabular}

the ablation or elimination of material from the layer is demonstrated. The EDX profiles provided by line scans analysis show the composition along the transversal section of the laser scribe. EDX line scans profiles are generated by the counts of electrons with different energies corresponding to different materials obtained from the scan of the electron beam from the SEM over a selected line in the sample. By the representation of these counts the profiles of the materials involved in the sample are obtained. This information is correlated with the amount of material present in the sample. Confocal topographical profiles show the height profile of the laser scribe, confirming the material removal by the laser scribing process. For ablation threshold calculations, a confocal topographical image has been used in order to measure the crater diameters (see Refs. $[8,9]$ for further details). In order to assess the heat affected zone in the second laser step, Raman spectra were run with a Renishaw Micro-Raman System. The deconvolution of Raman spectra that yields quantitative information regarding crystallinity can, thus, be correlated to the material damage by means of the heat affected zone generated during the laser scribing. In that sense, due to the high temperatures reached by the a-Si:H layer during the interconnection steps (P2 and P3), a heat affected zone, mostly associated with morphological changes induced in the material, could be generated around the ablated portion. In order to get this additional quantitative information, Raman crystallinity fraction, $\phi_{c}$, can be evaluated from the deconvoluted Raman spectra as the ratio of the area under the peaks related to crystalline parts over the total area of the silicon related peaks.

\section{Ablation thresholds calculation}

In laser selective ablation processes it is important to determine the appropriate energy density values that lead to effective material removal with minimum side effects. Ablation thresholds are very helpful for establishing limiting values for potential parametric windows. Ablation thresholds are obtained by measuring the increasing value of the ablation crater diameter as a function of the beam fluencies, a variable directly related to the ablation threshold $[8,9]$.

A summary of the threshold energy densities for single pulse ablation is presented in Table 1. The ablation thresholds correspond to single pulse radiation for the two different lasers and the materials which make up the solar cell. At $355 \mathrm{~nm}$ and $15 \mathrm{~ns}$ pulses the ablation thresholds tendency is similar to the ones obtained at $355 \mathrm{~nm}$ and $8 \mathrm{ps}$ pulses, except for the case of Asahi-U and the aluminium. This suggests that the effect of the pulsewidth is not so relevant for the temporal range in which the study is centred. In the case of aluminium and the high conductive TCO Asahi-U, the dependency with the pulsewidth is higher, given the absorption mechanisms involved in metals [10].

\section{Laser scribing processes}

The experiments have been carried out in unfinished solar cells, where each laser step has been assessed with the appropriate deposition of layers. For the first step (P1), a unique layer of transparent 
Table 2

Laser parameter for the optimal scribes of TCO's in terms of fluence $\left(\mathrm{J} / \mathrm{cm}^{2}\right)$ and number of pulses per location.

\begin{tabular}{|c|c|c|c|c|c|c|}
\hline \multirow[t]{2}{*}{ Materials } & \multicolumn{2}{|c|}{$\phi\left(\mathrm{J} / \mathrm{cm}^{2}\right)$} & \multicolumn{2}{|c|}{ No of pulses per location } & \multicolumn{2}{|l|}{$\phi\left(\phi_{\mathrm{th}}\right)$} \\
\hline & ns & ps & ns & ps & ns & ps \\
\hline Asahi-U $U^{(8)}\left(\mathrm{SnO}_{2}: \mathrm{F}\right)$ & 4.16 & 1.47 & 5 & 8.6 & $2.9\left(\phi_{\mathrm{th}}\right)$ & $3.5\left(\phi_{\mathrm{th}}\right)$ \\
\hline ITO $\left(\mathrm{SnO}_{2}: \operatorname{In}_{2} \mathrm{O}_{3}\right)$ & 0.43 & 0.79 & 1.3 & 1.1 & $2\left(\phi_{\mathrm{th}}\right)$ & $2.2\left(\phi_{\mathrm{th}}\right)$ \\
\hline $\mathrm{ZnO}: \mathrm{Al}$ & 0.85 & 1.2 & 2.2 & 1.7 & $4.6 .9\left(\phi_{\mathrm{th}}\right)$ & $3.7\left(\phi_{\mathrm{th}}\right)$ \\
\hline
\end{tabular}

conductor oxide (TCO) is deposited on glass. In the second step (P2), an additional layer of amorphous silicon was deposited on top of the TCO and for the last step (P3), an "ad-hoc" sample in substrate configuration with an $\mathrm{ZnO}: \mathrm{Al}$ (AZO) layer acting as back contact was developed for the evaluation of its removal.

\subsection{P1: TCO scribing}

For P1 three TCO's were evaluated: Asahi-U, ITO and AZO. Table 2 shows the results in terms of fluence and number of pulses necessary to carry out the process. Fig. 1 shows the SEM images of the scribes corresponding to the laser parameters shown in Table 2. SEM images were acquired in backscattering mode in order to obtain material information through the difference in contrast of the image, this difference is related with the difference of material and therefore with the elimination of the material in the middle of the groove. In Fig. 1 confocal profiles results are presented in a com- parative way, where the differences in morphology are enhanced to see the differences between the two pulsewidth more clearly.

According to the threshold obtained for optimal scribes, it was necessary more energy to perform the process in the case of Asahi$\mathrm{U}$ than for the other two. The overlap, i.e. the number of pulses per location, required to remove the complete layer of TCO was bigger in the case of Asahi-U, consistently again with the differences in threshold values. It must be pointed out the necessity of more energy to perform the scribe in the case of AZO than for ITO and also the difference in morphology (Fig. 1), although the ablation thresholds are the same. Further studies will be done in this subject.

For picosecond radiation the resulting scribes show less affectation in the edge, whereas in the case of nanosecond they present ridges at the border. SEM images illustrate similar morphology for the scribes produced by both pulses, but the confocal profiles reveal important differences at the border of the scribes in the case of Asahi-U and ITO. This is probably due to the fact that the process

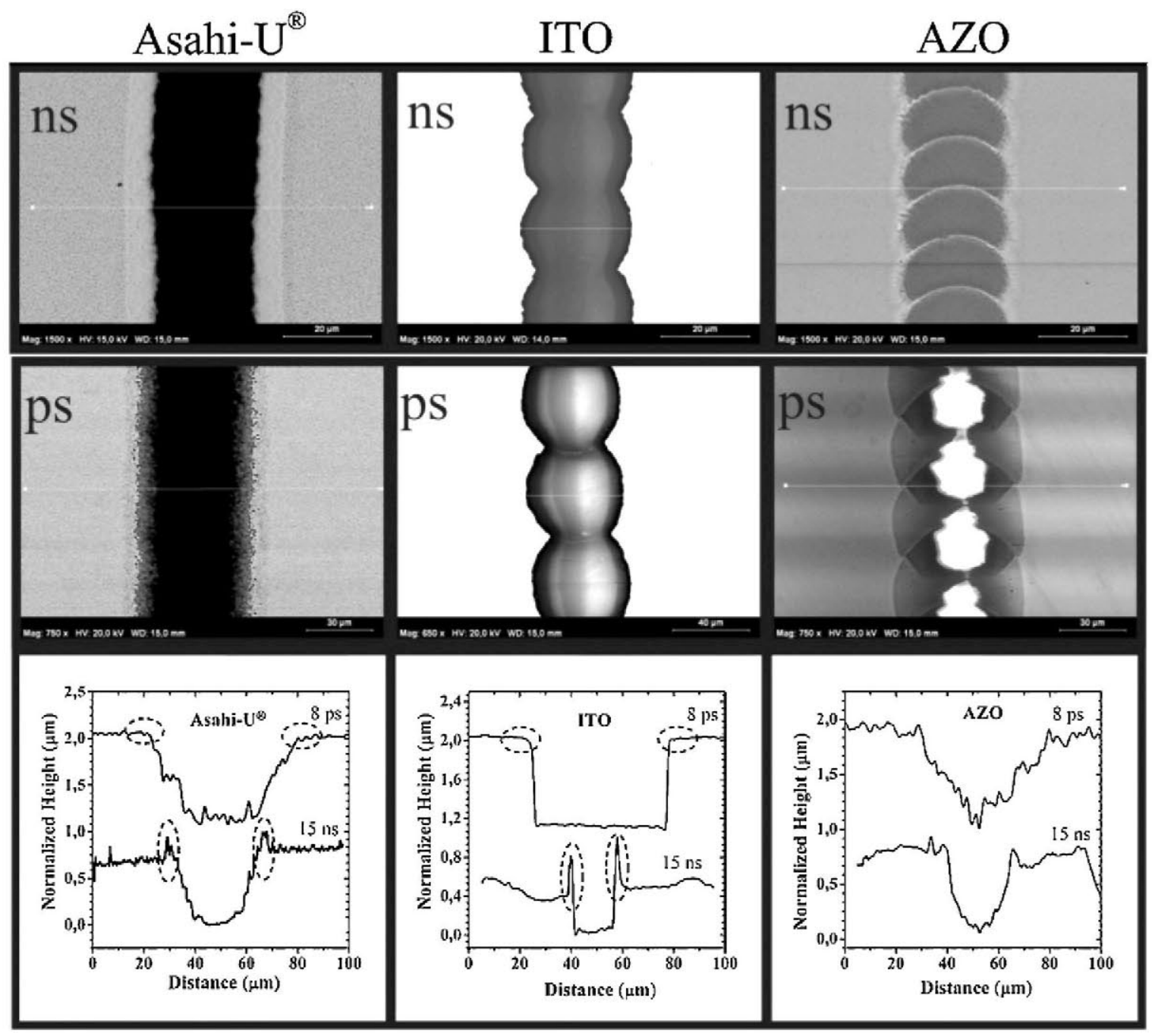

Fig. 1. SEM images and confocal profiles of laser scribes at nanosecond and picosecond radiation of three different TCO over glass. 
Table 3

Laser parameter for the optimal scribes of amorphous silicon structure layer in terms of fluence $\left(\mathrm{J} / \mathrm{cm}^{2}\right)$ and number of pulses per location.

\begin{tabular}{|c|c|c|c|c|c|c|}
\hline \multirow[t]{2}{*}{ Laser sources } & \multicolumn{2}{|c|}{$\phi\left(\mathrm{J} / \mathrm{cm}^{2}\right)$} & \multicolumn{2}{|c|}{ No of pulses per location } & \multicolumn{2}{|l|}{$\phi\left(\phi_{\mathrm{th}}\right)$} \\
\hline & ns & ps & ns & ps & ns & ps \\
\hline Low overlap & 0.59 & 1.26 & 1.6 & 1.8 & $5.1\left(\phi_{\mathrm{th}}\right)$ & $5.8\left(\phi_{\mathrm{th}}\right)$ \\
\hline High overlap & 0.47 & 0.57 & 3.6 & 7.9 & $4.1\left(\phi_{\mathrm{th}}\right)$ & $2.6\left(\phi_{\mathrm{th}}\right)$ \\
\hline
\end{tabular}
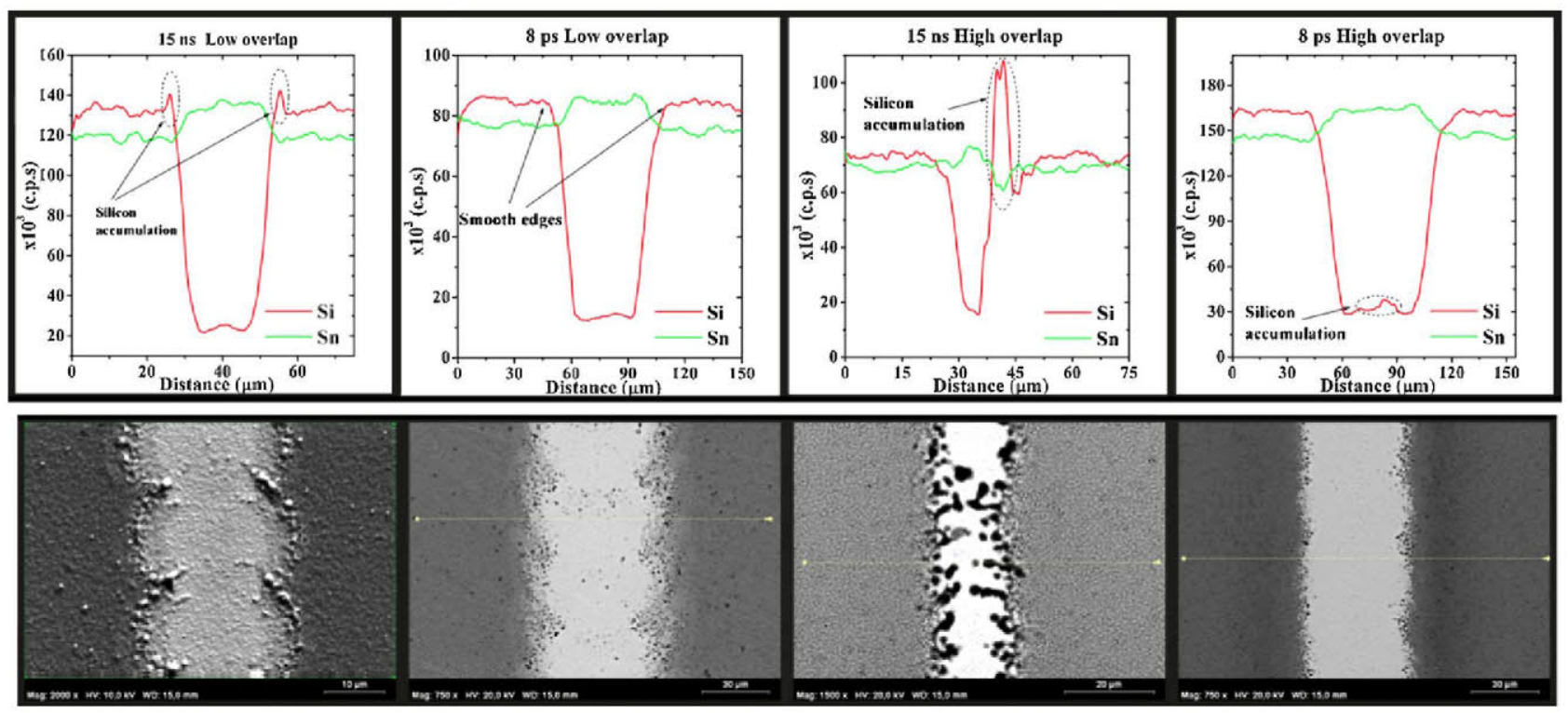

Fig. 2. SEM images and EDX profiles of laser scribes at nanosecond and picosecond radiation of a-Si:H(pin)/Asahi-U/glass.

of absorption for the distinct pulsewidths is different as it is widely discussed in literature [10]. Complete removal of the TCO on the groove has been proved by simple measurement of contact resistance and additional EDX profiles were obtained to confirm this fact. In terms of fluence, better morphological result obtained in ITO scribes could be explained by the necessity of less fluence and lower number of pulses to remove the layer than for the other TCO's. The results are consistent with previous works from the authors [6,7].

\subsection{P2: a-Si:H selective ablation}

The complete ablation of the amorphous silicon layer has been accomplished without damaging the underneath layer of TCO for both laser sources. This process has been assessed by EDX microanalysis, which allowed the evaluation of the selective removal of the silicon layer, additionally to the assessment of the damage on the TCO. In Table 3 are represented the laser parameters for the two different radiations, attending to the best result obtained in two different speed regimes, corresponding to different overlaps. It has been found that for picosecond radiation more energy is needed in the case of low overlap but more pulses are needed when the fluence is similar to the used with nanosecond pulses.

Nevertheless, it is more important in this case to assess the morphological aspects of the groove together with the removal of material and damage of the layer underneath. To accomplish that, Fig. 2 shows the best scribes obtained with nanosecond and picosecond radiation characterized with EDX spectroscopy and SEM images for laser parameters presented in Table 3 for low overlap in both cases.
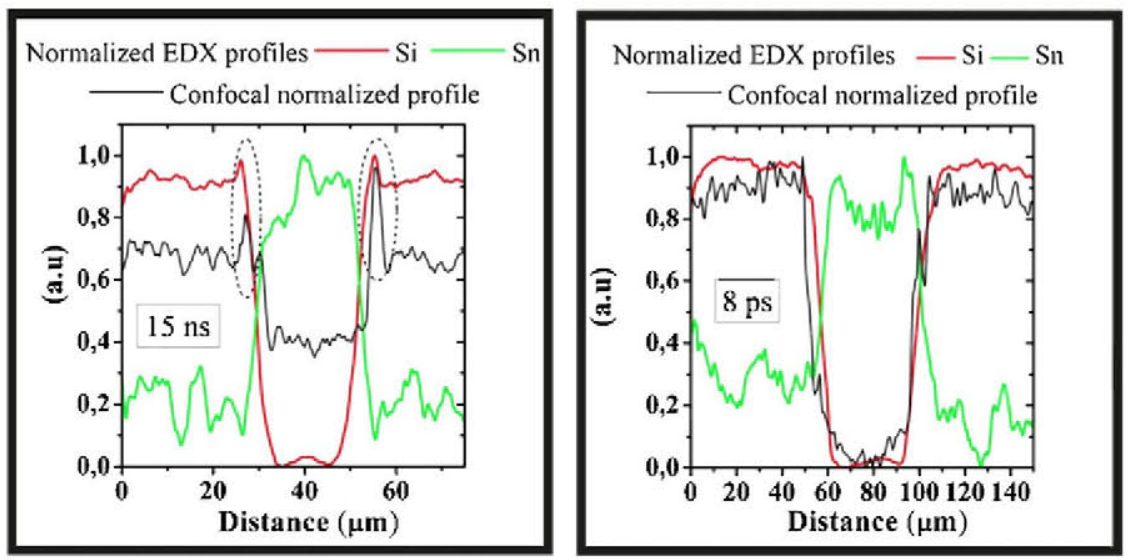

Fig. 3. Confocal and EDX profiles of laser scribes at nanosecond and picosecond radiation of a-Si:H(pin)/Asahi-U/Glass for optimal scribes. 

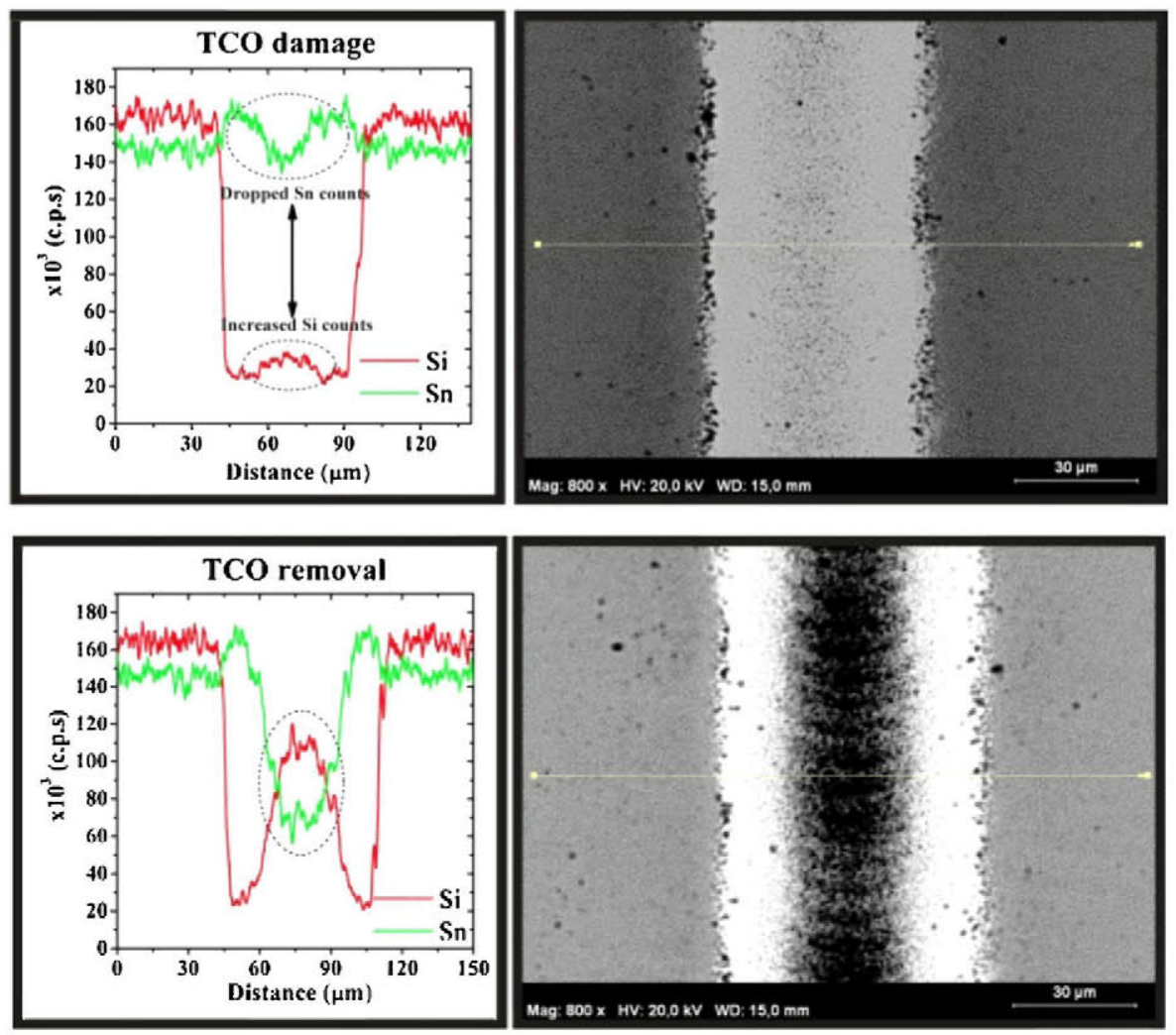

Fig. 4. EDX profiles and SEM images of laser scribes at nanosecond and picosecond radiation of a-Si:H(pin)/Asahi-U/glass to illustrate the damage on the TCO layer.

To correlate the silicon accumulation on the edge of the groove with its real height, the confocal and EDX profiles for the optimal scribes have been represented together as can be seen in Fig. 3. With EDX profiles it can be demonstrated selective ablation of silicon and also the damage of the TCO layer by the drop in the atom counts coming from silicon and tin related to the two different layers as Fig. 4 reveals. When the silicon profile drops in the middle of the groove, without decreasing the counts coming from the tin of the Asahi-U in the same location, this indicates that the amorphous silicon layer has been removed without damaging the layer underneath. To illustrate this in a better way, Fig. 4 shows the damage on the TCO layer during the P2 process, in EDX profiles. When the counts coming from the TCO drop in the middle of the groove, it is seen an associated increase in the counts of silicon appearing at the same position due to the silicon contribution of the glass. This becomes more evident when more energy is deposited. When the silicon counts exceed the ones from the tin, this means that there is a large contribution of the silicon coming from the glass, implying that the TCO layer has been removed. This assessment is especially relevant because the damage of the TCO layer leads to an increase in the series resistance of the final device.

\subsection{P3: back contact selective ablation}

Finally, the last step to obtain the laser monolithic interconnection from the film side is the laser scribe isolation of the back contact. In this case the study has been carried out on AZO as back contact. The achievement of the back contact selective ablation from the film side would offer the possibility of using just one laser source in the production line and would suggest the feasibility of applying this approach to substrate configurations and to the use of non-transparent substrates.

Complete removal of AZO layer over the silicon structure was achieved for the two pulsewidths and the process parameters are collected in Table 4. The optimal scribes are shown in Fig. 5 for nanosecond and picoseconds radiation. These figures illustrate the selective ablation achievement of the TCO layer over the stack of the cell. The scribes for both pulsewidth are free of AZO at its bottom confirmed by the EDX profiles. Confocal profiles show there is no presence of ridges on the edge of the grooves.

By comparing the results at nanosecond and picosecond in Fig. 5 , it is shown that the overall morphological quality of the grooves obtained with both sources is similar, but paying attention to the Confocal profiles and the topographical images it is seen that the quality of the groove for nanosecond radiation is slightly better, since the Gaussian profile of the laser beam on the edges of the grooves is more pronounced in the case of picoseconds radiation, due to its bigger diameter.

Raman spectroscopy characterization has been performed for this laser step, in order to evaluate the state of the remaining silicon layer after irradiation. The damage of the silicon layer is assessed with the variation of the crystalline fraction resulting from the Raman spectra along the groove. The increase of crystalline fraction entails a microstructural modification of the intrinsic material,

Table 4

Laser parameter for the optimal scribes of $\mathrm{P} 3$ step over $\mathrm{ZnO}: \mathrm{Al}(\mathrm{AZO})$ layer of the pseudo device in terms of fluence $\left(\mathrm{J} / \mathrm{cm}^{2}\right)$ and number of pulses per location.

\begin{tabular}{|c|c|c|c|c|c|c|}
\hline \multirow[t]{2}{*}{ Laser sources } & \multicolumn{2}{|c|}{$\phi\left(\mathrm{J} / \mathrm{cm}^{2}\right)$} & \multicolumn{2}{|c|}{ No. of pulses per location } & \multicolumn{2}{|l|}{$\phi\left(\phi_{\text {th }}\right)$} \\
\hline & $\mathrm{ns}$ & ps & ns & ps & ns & ps \\
\hline & 0.71 & 0.96 & 1.03 & 1.04 & $3.8\left(\phi_{\mathrm{th}}\right)$ & $3.0\left(\phi_{\mathrm{th}}\right)$ \\
\hline
\end{tabular}




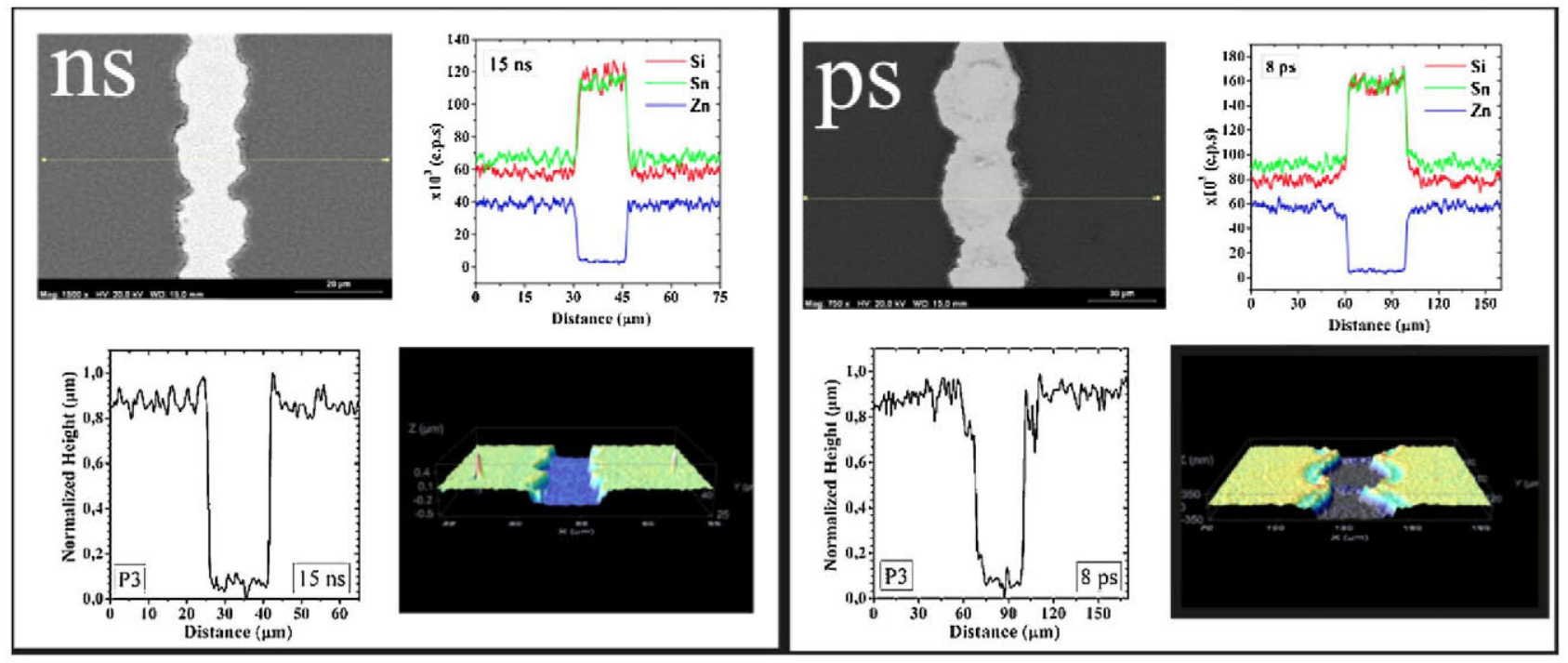

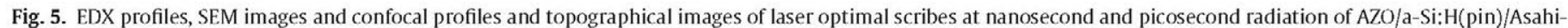
U/glass.
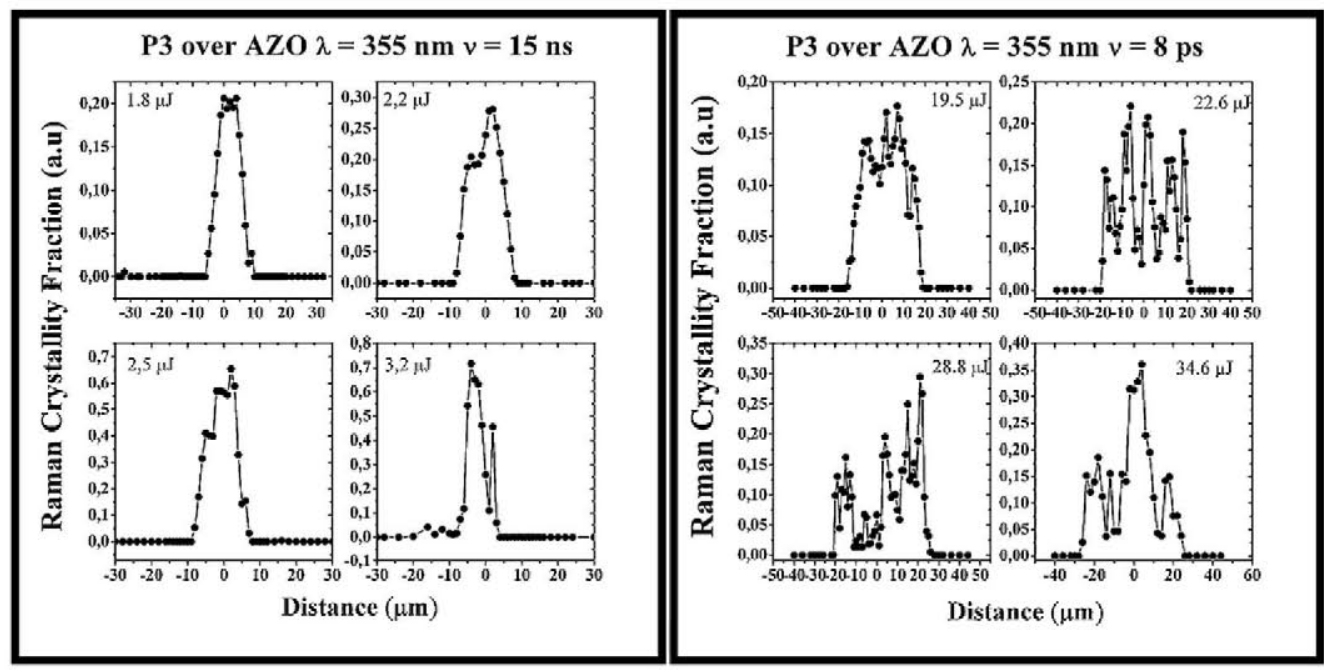

Fig. 6. Raman crystallinity fraction of $\mathrm{P} 3$ process performed with nanosecond and picoseconds radiation.

mainly related to thermal effects. On this basis, these alterations of the remaining silicon could result in the reduction of the intrinsic material electrical resistance [1]. The study has been made for the optimal speed of process, increasing the energy to evaluate the state of the remaining material. Fig. 6 shows the result for the two pulsewidths. Particularly remarkable are the low values obtained for the crystalline fraction; less than 0.2 in the lower energies for both pulse durations. Comparing these results with the ones obtained by laser back-scribing performed at $532 \mathrm{~nm}$ [11], the crystalline fraction generated with $355 \mathrm{~nm}$ just in the middle of the groove is considerably lower than at the edge of the ablated groove with $532 \mathrm{~nm}$, where the values of the fraction reach up to 0.8 .

Further experiments should be carried out in TCO back contact for complete solar cells in substrate configuration, in order to obtain electrical measurements, and assess completely the process.

\section{Conclusion}

A comparative study of laser monolithic interconnection with nanosecond and picosecond pulses has been presented. Ablation thresholds show consistent results with the scribing process, for which it has been shown the differences in morphology terms between nanosecond and picosecond scribes. The achievement of the complete selective ablation from the film side, without removal of bottom layers of the a-Si solar cell, and especially the good results obtained in the case of selective removal of AZO with UV ns radiation opens the possibility to develop industrially the three laser steps of monolithic interconnection from the film side with the laser sources presented here, whilst the usual practice is to carry out P2 and P3 from the glass side. Additionally, very good results were obtained of Raman crystalline fraction for the remaining silicon after P3 laser step performed in direct scribing configuration. It must be emphasized that the process has been assessed with "ad hoc" samples for the ablation process evaluation. Further experiments must be focused in a deep study of this laser interconnection on real and complete devices.

\section{Acknowledgement}

This work has been financed by the Spanish National R\&D Plan under CLASICO ENE2007-67742-C04 and PSE-MICROSIL08. 


\section{References}

[1] W. Shinohara, M. Shima, S. Taira, K. Uchihashi, A. Terakawa, Applications of laser patterning to fabricate innovative thin film silicon solar cells, Proc. SPIE 6107 (2006), pp. 61070J1-61070J18.

[2] B. Rech, H. Wagner, Potential of amorphous silicon for solar cells, Appl. Phys. A 69 (1999) 155-167

[3] S. Golay, J. Meier, S. Dubail, S. Fay, U. Kroll, ShahF A., First pin/pin micromorph modules by laser patterning, in: Proceedings of the 16th EU Photovoltaic Solar Energy Conference, Glasgow, 2000.

[4] S.R. Praschek, W. Riedl, H. Hoermann, H.G. Goslowsky, Laser patterning of a-Si solar modules with transparent conducting zinc oxide back electrodes. Photovoltaic specialists' conference, in: Conference Record of the Twenty Second IEEE, 2, 1991, pp. 5-1289.

[5] H.J. Booth, Recent applications of pulsed lasers in advanced materials processing, Thin Solid Films (2004), 453-454, pp.s 450-457.

[6] C. Molpeceres, S. Lauzurica, J.L. Ocana, J.J. Gandia, L. Urbina, J. Carabe, Microprocessing of ITO and a-Si thin films using ns laser sources, ]. Micromech. Microeng. 15 (2005) 1271-1278.
[7] C. Molpeceres, S. Lauzurica, J.J. García-Ballesteros, M. Morales, G. Guadaño, J.L. Ocaña, S. Fernández, J.J. Gandía, F. Villar, O. Nos, J. Bertomeu, Selective ablation of photovoltaic materials with UV laser sources for monolithic interconnection of devices based on a-Si:H, Mater. Sci. Eng. B 159-160 (2009) 18-22.

[8] J.M. Liu, Simple technique for measurements of pulse Gaussian-beam spot sizes, Opt. Lett. 7 (1982) 196.

[9] S. Preuss, A. Demchuk, M. Stuke, Sub-picosecond UV laser ablation of metals, Appl. Phys. A 61 (1995) 33-37.

[10] B.N. Chichkov, C. Momma, S. Nolte, F. von Alvensleben, A Tünnermann, Femtosecond, picosecond and nanosecond laser ablat ion of solids, Appl. Phys. A 63 (1996) 109-115.

[11] M. Colina, C. Molpeceres, M. Holgado, J. Gandia, O. Nos, J.L. Ocaña, Study of the refractive index change in a-Si:H thin films patterned by $532 \mathrm{~nm}$ laser radiation for photovoltaic applications, Thin Solid Films (2010), doi:10.1016/j.tsf.2010.04.016. 